\title{
Plasma-induced selectivity in bone cancer cells death
}

Dr. Cristina Canal ${ }^{1,2 *}$, Msc. Raul Fontelo ${ }^{1}$, Bsc. Ines Hamouda ${ }^{1}$, Dr. Jordi GuillemMarti $^{1,2}$, Prof. Uros Cvelbar ${ }^{3}$, Prof. Maria-Pau Ginebra ${ }^{1,2,4}$

${ }^{1}$ Biomaterials, Biomechanics and Tissue Engineering Group, Dpt. Materials Science and Metallurgy, Technical University of Catalonia (UPC), c. Eduard Maristany 10-14, 08019 Barcelona, Spain;

${ }^{2}$ Barcelona Research Center in Multiscale Science and Engineering, Universitat Politècnica de Catalunya, Barcelona, Spain

${ }^{3}$ Department of Surface Engineering and Optoelectronics (F-4), Jožef Stefan Institute, Jamova cesta 39, 1000 Ljubljana, Slovenia

${ }^{4}$ Institute for Bioengineering of Catalonia, c/ Baldiri i Reixach 10-12, 08028 Barcelona, Spain

*corresponding author: cristina.canal@upc.edu

Tel. +34934017810

Mailing address: Biomaterials, Biomechanics and Tissue Engineering Group, Dpt.

Materials Science and Metallurgy, Technical University of Catalonia (UPC), c. Eduard Maristany 10-14, 08019 Barcelona, Spain

cristina.canal@upc.edu

Tel. +34934017810 


\section{Abstract}

BACKGROUND: Current therapies for bone cancers - either primary or metastatic - are difficult to implement and unfortunately not completely effective. An alternative therapy could be found in cold plasmas generated at atmospheric pressure which have already demonstrated selective anti-tumor action in a number of carcinomas and in more relatively rare brain tumors. However, its effects on bone cancer are still unknown. METHODS: Herein, we employed an atmospheric pressure plasma jet (APPJ) to validate its selectivity towards osteosarcoma cell line vs. osteoblasts \& human mesenchymal stem cells. RESULTS: Cytotoxicity following direct interaction of APPJ with cells is comparable to indirect interaction when only liquid medium is treated and subsequently added to the cells, especially on the long-term (72h of cell culture). Moreover, following contact of the APPJ treated medium with cells, delayed effects are observed which lead to $100 \%$ bone cancer cell death through apoptosis (decreased cell viability with incubation time in contact with APPJ treated medium from 24h to $72 \mathrm{~h}$ ), while healthy cells remain fully viable and unaffected by the treatment. CONCLUSIONS: The high efficiency of the indirect treatment indicates that an important role is played by the reactive oxygen species (ROS) and reactive nitrogen species (RNS) in the gaseous plasma stage and then transmitted to the liquid phase, which overall lead to lethal and selective action towards osteosarcoma cells. These findings open new pathways for treatment of metastatic bone disease with a minimally invasive approach.

Keywords: atmospheric pressure plasma jet; bone cancer; Osteosarcoma; osteoblasts; SaOS-2; hMSC; HOb; liquids; 


\section{Introduction}

It is estimated that only in year 2016 alone, more than 3,600 new cases of bone cancers will be diagnosed in the USA, with $50 \%$ mortality rate ${ }^{1}$. Malignancies in the bone can be of different origin. Either the cancer cells originate from the bone tissue or the bone marrow itself (primary bone cancers), or are metastasized from a tumor that originated elsewhere in the body (secondary bone cancers). In 2008, approximately 280,000 adults in USA were living with metastatic bone disease, with $68 \%$ of cases occurring in patients with primary breast, prostate or lung cancer ${ }^{2}$.

Most common treatment options for bone cancer include surgery, chemotherapy and radiation therapy or their combinations, depending on the kind and extension of the cancer. Recently the gene-targeted therapies have received more attention along with other local therapies such as laser ablation, hyperthermia, cryosurgery, and thermal plasma coagulation. In all cases, the therapies are very difficult to perform and not completely effective ${ }^{3}$. Additionally, the agents used for example in systemic chemotherapy have many undesirable side effects which include the change of the normal skeletal development and bone remodeling causing more resorption, leading to a loss in bone mass, reduced strength and a higher risk of bone fractures in both disease-free and bone metastasis patients ${ }^{3}$.

Cold gaseous plasmas created with electrical discharges are a source of a vast number of reactive species (molecules, atoms, ions, electrons, photons, UV \& visible radiation) which can affect eukaryotic cells through complex biochemical procedures. One key aspect, which has attracted attention in the novel area of plasma medicine recently named "plasma oncology", is the ability of cold atmospheric pressure plasmas (APP) to 
selectively kill cancer cells without damaging the surrounding tissues. APP formed in air generates reactive oxygen (ROS) and nitrogen species (RNS). These species appear to induce cell cycle arrest at relatively low plasma “doses”, lead to apoptosis at higher doses ${ }^{4}$, and ultimately cause necrosis at the highest doses ${ }^{5}$. Furthermore, a remarkable similarity of anti-tumor action for all types of cancers investigated was observed: carcinomas, skin cancer and brain tumors. This discovery was reported in vitro and in vivo for different non-thermal plasmas, where studies were conducted on a variety of cancer types ${ }^{6}$ including melanoma ${ }^{7}$, cervical cancer ${ }^{8}$, hepatocellular carcinoma, breast carcinoma ${ }^{9}$, papilloma, colon cancer, glioblastoma, etc. ${ }^{6}$.

In general, during APP treatment cells (or tissues) are covered by liquids, so the role of the plasma-induced reactive species in liquids is being investigated as an indirect potential treatment and to ascertain the role of this liquid phase during APP treatments. In fact, the generation of APP jets in close vicinity to liquid media under certain conditions can lead to the formation of a variety of species, among which ROS and RNS. In particular, not only the gas phase, but also the components of the liquid media (water, aqueous solutions or culture media) determine the species generated. For instance, in plasma-treated aqueous systems different reactive species have been detected ${ }^{10}$; Short-lived transient species (peroxynitrite $\mathrm{ONOO}^{-}$, hydroxyl radical $\bullet \mathrm{OH}$, nitrogen dioxide $\bullet \mathrm{NO}_{2}$, nitric oxide •NO, radicals and superoxide $\mathrm{O}_{2}^{-}$) and long-lived chemical products (ozone $\mathrm{O}_{3}$, hydrogen peroxide $\mathrm{H}_{2} \mathrm{O}_{2}$, nitric acid $\mathrm{HNO}_{3}$, nitrous acid $\mathrm{HNO}_{2}$ ) are formed in water following a plasma discharge. APP is known to produce various active neutral short and long living ROS in cell culture medium $\left(\bullet \mathrm{OH}, \mathrm{O} \bullet\right.$, excited oxygen $\mathrm{O}(1 \mathrm{D}), \mathrm{O}_{2}(1 \Delta \mathrm{g})$ and $\mathrm{O}_{3}, \mathrm{HO}_{2}$ and $\left.\mathrm{H}_{2} \mathrm{O}_{2}\right)^{11}$. Peroxinitrite chemistry was also shown to significantly participate in the biological effects of APP activated media, by mediating the formation of $\mathrm{NO}_{2}^{-}, \mathrm{NO}^{-}$, and 
- $\mathrm{OH}$ radicals and $\mathrm{NO}^{+}$ions through post-discharge processes in plasma-activated liquid ${ }^{5}$. Different authors have shown that APP treated media has anti-tumor effects against a variety of cancer cells (ie. Glioblastoma ${ }^{12,13}$, ovarian ${ }^{14,15}$, gastric $^{16}$, pancreatic ${ }^{17}$, lung $^{18}$ or colorectal carcinoma ${ }^{19}$ ), though the role of the liquid phase in the anticancer action of APP is not fully understood yet ${ }^{5}$.

In spite of the recent progress in the area, the prospective effects of plasmas for bone cancer therapy are still unknown. Components of the complex bone microenvironment contribute significantly to the growth and proliferation not only of primary cancers, such as osteosarcoma or myeloma, but also to the process of metastasis of epithelial-derived cancers such as prostate or breast cancer ${ }^{3}$.

The aim of our work is to evaluate the potential of APP for bone cancer therapy and elucidate the mediation of liquid media in the process. The prospective of employing APP-treated liquids opens new potential pathways for treatment of metastatic bone disease with a minimally invasive approach. In this paper, an atmospheric pressure plasma jet (APPJ) and its effects on the cell culture media are analyzed. Its effects are evaluated either directly on cell cultures or indirectly through liquid media treatment of bone cancer cells (human osteosarcoma) as well as on healthy cells (human mesenchymal stem cells).

\section{MATERIALS AND METHODS}


Sarcoma osteogenic (SaOS-2) cells (ATCC, USA) were cultured in McCoy’s 5A (Sigma) supplemented with 10\% fetal bovine serum (FBS), 2 mM L-glutamine, penicillin/ streptomycin (50 U/ml and $50 \mu \mathrm{g} / \mathrm{ml}$, respectively), and 1\% sodium pyruvate, all from Invitrogen. Cells from passages 23-29 were used in all experiments.

Human mesenchymal stem cells (hMSCs, Millipore, USA) were expanded in Advanced DMEM supplemented with 10\% (FBS), 2 mM L-glutamine, penicillin/streptomycin (50 $\mathrm{U} / \mathrm{ml}$ and $50 \mu \mathrm{g} / \mathrm{ml}$, respectively), all from Invitrogen. Cells from passages 3-4 were used in all experiments.

Human Osteoblasts (HObs, Cell applications inc., USA) were expanded in HOb medium (Cell applications inc., USA). Cells from passage 2 were used in all experiments.

\section{Methods}

\section{Plasma Treatment}

An atmospheric pressure plasma jet was created using He (5.0 Linde, Spain) as plasma carrier gas in a jet design with a single electrode as described elsewhere ${ }^{20}$. The discharge electrode was a copper wire with a diameter of $0.1 \mathrm{~mm}$ inserted inside of a $1.2 \mathrm{~mm}$ inner diameter quartz capillary tube, covered by a polytetrafluoroethylene (PTFE) holder. The electrode was connected to a commercial high voltage power supply from Conrad Electronics (nominally $6 \mathrm{~W}$ power consumption). The discharge was operating with sinusoidal waveform at $25 \mathrm{kHz}$ with (U) $\sim 2 \mathrm{kV}$ and (I) $~ 3 \mathrm{~mA}$. Helium flow in the capillary was regulated between 1 and $5 \mathrm{Ln} / \mathrm{min}$ through a Bronkhorst MassView flow controller. 
To characterize the plasma effects on the liquids, $4 \mathrm{~mL}$ of the corresponding cell culture medium were placed under the jet at different distances from 1 to $20 \mathrm{~mm}$ from the tube opening with electrode to the surface. The liquid was placed in a Petri dish of $30 \mathrm{~mm}$ in diameter, whereas treatment times where changed from 5 to $30 \mathrm{~min}$.

\section{Optical Emission Spectroscopy (OES)}

OES was used to determine the main plasma emitting species. The equipment used was a spectrometer F600-UVVIS-SR (2m-StellarNet Inc.), which was connected to an optical fiber (QP600-2SR-Ocean Optics) with lens that collected information from the measure point near the plasma jet. For data processing the SpectraWiz software (StellarNet Inc.) was used.

The optical fiber was placed perpendicular to the jet and measurements were made at two different positions: at the beginning of the jet (plasma, designated as distance $d=0$ ) and about ten millimeters below (in the post-discharge, $d=10 \mathrm{~mm}$ ). All results were obtained with an integration time of $1000 \mathrm{~ms}$ and an average of 10 scans.

\section{Characterization of liquid media}

For the characterization of the liquid media, each parameter was measured immediately after the APPJ treatment. $\mathrm{pH}$ and conductivity were measured using an MM 41 Crison multimeter with 5028 and 5070 probes, respectively.

The concentration of $\mathrm{H}_{2} \mathrm{O}_{2}$ was measured with semiquantitative test strips (Quantofix Peroxides test sticks; Sigma-Aldrich) according to the manufacturer's instructions. The same method was used for $\mathrm{NO}_{2}{ }^{-}$and $\mathrm{NO}_{3}{ }^{-}$(Quantofix Nitrites and Nitrates test strips; Sigma-Aldrich). 


\section{Cell culture}

Both types of cells were seeded in their corresponding medium and maintained at $37^{\circ} \mathrm{C}$ in a humidified atmosphere containing 5\% $\mathrm{CO}_{2}$. Subconfluent cells were trypsinized, centrifuged and seeded in a 96-well plate at a density of 10,000 cells/well, and incubated in $100 \mu \mathrm{l}$ of complete medium for $24 \mathrm{~h}$.

\section{Cell Viability Assay}

Two different effects of APPJ treatment were evaluated for both cell cultures: direct and indirect treatment. For the direct treatment, each well was seeded with 10,000 cells $24 \mathrm{~h}$ prior to treatment, and was replaced with $150 \mu \mathrm{L}$ of fresh medium right before treatment (McCoy for SaOS-2 cells and advDMEM for hMSC and HOb cells). Plasma treatment was performed directly on this medium with adherent cells using atmospheric He plasma gas flow rate of $5 \mathrm{Ln} / \mathrm{min}$, at $20 \mathrm{~mm}$ distance between the end tip of the capillary and the medium surface. Treatments were performed for different times ranging from $30 \mathrm{~s}$ to 90 S.

Alternatively, the indirect treatment was performed by applying APPJ only to $4 \mathrm{ml}$ of culture medium placed in $30 \mathrm{~mm}$ diameter Petri dishes. Medium was incubated for two hours at $37^{\circ} \mathrm{C}$ before the treatment. After incubation, each Petri dish containing either McCoy for SaOS-2 cells or advDMEM for hMSC and HOb cells was treated with APPJ at the flow rate $5 \mathrm{~L} / \mathrm{min}$, where the distance between the beginning of the plasma jet and the medium surface was constant at $20 \mathrm{~mm}$. Treatment times were 10, 20 and $30 \mathrm{~min}$. Then, $150 \mu \mathrm{L}$ of plasma treated medium were immediately transferred to 96 well plates containing 10.000 cells that had been seeded 24 hours prior the plasma medium treatment. 
The treatment times of direct and indirect treatment were selected on the basis of the surface ratio of the wells employed in each treatment (eq. 1):

$t_{\text {indirect treatment }}=\frac{\text { Surface petri dish }}{\text { Surface well }(96 \text { well plate) }} t_{\text {direct treatment }}$

The plates were incubated at $37^{\circ} \mathrm{C}$ for $24 \mathrm{~h}, 48 \mathrm{~h}$ and $72 \mathrm{~h}$ for further evaluation of cell viability. For both direct and indirect treatments, a negative control with no cells and only untreated culture medium and a positive control with the corresponding cell type in untreated medium were evaluated as well. This positive control was employed as reference for $100 \%$ cell viability.

After each incubation period, cells were lysed using $100 \mu \mathrm{L}$ of Mammalian Protein Extraction Reagent (M-PER, Thermo-Scientific). The lysates were analyzed to quantify the number of cells by measuring the lactate dehydrogenase activity (Citotoxity Detection Kit, LDH; Roche Applied Science). Absorbances were measured spectrophotometrically at $492 \mathrm{~nm}$ using a Synergy HTX multi-mode microplate reader (BioTek).

Flow cytometry

SaOS-2 cells were seeded at $8 . \times 10^{4}$ cells/well density in 24 well plates and incubated for $24 \mathrm{~h}$. Then, $1 \mathrm{~mL}$ of McCoy medium treated with APPJ for $30 \mathrm{~min}$ at $5 \mathrm{Ln} / \mathrm{min}$, and 20 mm distance using the same conditions described above, was transferred onto the adhered cells. After 24, 48 or $72 \mathrm{~h}$ the supernatants were collected, cells were tripsynized (TrypLE, Invitrogen) and centrifuged, and then stained with Alexa Fluor 488 Annexin V 
and propidium iodide (PI) following the supplier's instructions (Vybrant apoptosis assay kit, Molecular probes). Apoptotic, necrotic and healthy cells were analysed in a Gallios multi-color flow cytometrer instrument (Beckman Coulter, Inc, Fullerton, CA) set up with the 3-lasers 10 colors standard configuration. Excitation was done using a blue (488nm) laser. Forward scatter (FS), side scatter (SS), green fluorescence (525/40nm) from Annexin V and red fluorescence (695/30nm) emitted by PI were collected using logarithmic scales. FS was used as discriminating parameter.

\section{Cell morphology}

Cells treated as described in the flow cytometry assay were fixed in 4\% paraformaldehyde (Sigma-Aldrich) for 30 min. After washing thrice with PBS, cells were permeabilized with Triton X-100 in PBS for 20 min. Permeabilized cells were washed thrice with 20 mM glycine in PBS (washing buffer) and blocked with 1\% BSA in PBS for 30 min. Then, cells were incubated with mouse anti-vinculin (1:100 in PBS; Invitrogen) for $1 \mathrm{~h}$ and rinsed thrice with washing buffer. Afterwards, cells were incubated with Alexa Fluor 488 goat anti-mouse (1:1000; Invitrogen) and Alexa Fluor 546 Phalloidin (1:300; Invitrogen) for $1 \mathrm{~h}$ in the dark. Thereafter, cells were washed thrice in washing buffer and nuclei were counterstained with DAPI (1:1000; Invitrogen). Finally, samples were mounted in Mowiol 4-88 (Invitrogen) before visualizing in a DM6000 fluorescence microscope (Leica Microsystems). Images were taken using a LAS AF7000 software (Leica Microsystems).

\section{Statistics}


Statistical differences were determined using one-way ANOVA with Tukey's post-hoc tests using Minitab 16 software (Minitab, Inc., USA). Statistical significance was considered when $\mathrm{p}<0.05$. Data are presented as mean \pm standard deviation.

\section{Results}

Characterization of the plasma jet

An APPJ created in pure He gas flow was employed to sustain the plasma discharge due to its low breakdown voltage. The free standing jet coming out of the capillary tube was exposed to open surrounding air at different He flow rates (Figure 1). The APPJ became destabilized at flow rates above $7 \mathrm{~L} / \mathrm{min}$. Conversely, the presence of liquid media allowed stabilization the jet and clearly modified its characteristics; as the liquid acted as a virtual ground or a counter electrode and helped sustaining plasma up to higher flow rates of $10 \mathrm{~L} / \mathrm{min}$ (Figure 1 ).

To investigate the species generated by the plasma expanding into air and interacting with the liquid phase and the cells below it, the gas phase of the jet was characterized by OES (Figure 2) at $10 \mathrm{~mm}$ distance from the exit of the capillary. This allowed the optimal collection of emission spectrum which was dominated by Helium peaks and molecular bands of mainly second positive $\mathrm{N}_{2}$ system, and first negative $\mathrm{N}_{2}{ }^{+}$system. Due to interactions with the surrounding environment $\mathrm{O} \bullet$ and $\bullet \mathrm{OH}$ radicals were detected as well.

The evolution of the different plasma species with increasing flow rates (1 to $8 \mathrm{~L} / \mathrm{min}$ ) was characterized for the free standing jet or during the treatment of a liquid cell culture medium (Figure 3). From these graphs the differences in the generated species are noted. 
More particularly in the free standing jet, the peak intensity of $\mathrm{N}_{2} 2^{\text {nd }}$ positive and $\bullet \mathrm{OH}$ are maximum at $2 \mathrm{Ln} / \mathrm{min}$ gas flow rate, whereas, $\mathrm{N}_{2}{ }^{+} 1^{\text {st }}$ negative steeply increases and saturates at higher flow rates. On the other side $\mathrm{N}_{2} 2^{\text {nd }}$ positive and $\bullet \mathrm{OH}$ decrease slowly going to higher flow rates. Similar maxima in intensity is then observed for all these 3 species during the treatment of liquid media at $2 \mathrm{~L} / \mathrm{min}$, whereas $\mathrm{N}_{2}{ }^{+} 1^{\text {st }}$ negative emission seems to be reduced by reaction with the medium at flow rates higher than $2 \mathrm{~L} / \mathrm{min}$. On the contrary, after $3 \mathrm{~L} / \mathrm{min}$ and past the maximum at $2 \mathrm{~L} / \mathrm{min}$, we observe a slow but steady increase for all species towards higher flow rates. At relatively high flow rates convection of the liquid media is observed (Figure 1), leading to more homogeneous distribution of species in the liquid medium. Therefore, given the potential relevance of the Reactive Oxygen (ROS) and Nitrogen species (RNS) in cancer treatment and stability of the APPJ system, we selected $5 \mathrm{~L} / \mathrm{min}$ for further experiments.

\section{Characterization of the liquid phase}

In order to unravel how APPJ influences the characteristics of the cell culture medium (McCoy's) employed for culturing of SaOS-2 cells, we investigated different parameters and resolved species created inside the liquid. This research encompassed changes of $\mathrm{pH}$ level, conductivity, and reactive species represented by peroxides $\left(\mathrm{H}_{2} \mathrm{O}_{2}\right)$, nitrates $\left(\mathrm{NO}_{2}{ }^{-}\right.$ ) and nitrites $\left(\mathrm{NO}_{3}{ }^{-}\right)$, where also different distances from the tip of the APPJ to the liquid surface were taken under consideration to optimize the treatment (Figure 4).

$\mathrm{pH}$ was shown to be fairly constant, while media conductivity tended to slowly increase with treatment time. Here it is worth noticing that this cell culture media didn't contain any buffers to avoid masking the possible effects of plasma. On the one hand, in all conditions tested, the plasma jet generates peroxides, nitrates and nitrites, and longer 
treatment times are related with higher concentrations. On another hand, the distance between the jet and the liquid media didn't seem to be a critical parameter.

Lower gas flows (ie. $2.5 \mathrm{~L} / \mathrm{min}$ ) were investigated (not shown) and their effects on the different liquid media parameters were shown to be either equivalent $(\mathrm{pH})$ or milder (regarding conductivity, and concentration of peroxides, nitrites and nitrates) than the results presented in Figure 4.

As result of these findings, $5 \mathrm{~L} / \mathrm{min}$ of He flow and $20 \mathrm{~mm}$ distance were selected and employed for treatment of McCoy's culture medium for the culture of SaOS-2 cells. When applying the same treatment to advanced Dulbeccos Modified Eagle Media (advDMEM), a similar trend was observed. As for McCoy's, $\mathrm{pH}$ remained largely unchanged, while increasing plasma treatment times led to increased conductivity, as well as the concentrations of nitrites which displayed slightly lower but comparable values (Supplementary material). The concentration of peroxides was found higher in advDMEM. For easier comparison, the same treatment was chosen for the two cell culture media for the indirect interaction study.

\section{In vitro biological effects}

The effects of plasma treatment were evaluated on both cancerous SaOS-2 cells and on healthy hMSC. Direct treatment of cells seeded in cell culture plates and covered with cell culture media (Figure 5) initially revealed similar reduction in cell viability (around $65 \%$ for both cancer and healthy cells 24 hours after the treatment). Cell culture for longer times (72h) revealed delayed effects of the plasma treatment: while SaOS-2 cells showed progressive increase in cell death related with the longest plasma treatment times (Figure 
5a), hMSCs were capable of recovering from the plasma treatment and 3 days later showed $100 \%$ of cell viability as compared to untreated hMSCs (Figure 5b). In contrast, HObs were unaffected with short plasma treatment and their proliferation was even stimulated at the longest time of direct treatment (1.5 min).

To ascertain the influence of the modifications produced in the liquid media by the plasma treatment, the indirect effects of APPJ were evaluated by treating cell culture media and then transferring this cell culture media to cultured SaOS-2, hMSC and HOb cells (Figure 6). Very clear effects were found. As observed with the direct treatment, contact of the cells with plasma treated cell culture media led to reduced cell viability after $24 \mathrm{~h}$, especially for cancer cells. Further incubation up to $72 \mathrm{~h}$ showed progressive reduction in SaOS-2 cell viability, with complete cell death for the samples treated for the longest time (30 min). In contrast, hMSC displayed better cell viability, with a minor decrease ( $\approx 90 \%$ of cell viability at $24 \mathrm{~h}$ as compared to cells cultured in untreated cell culture medium) for the longest plasma treatment time, which was recovered with incubation time. Similarly, healthy HOb cells treated for 10 and 20 min did not show any decrease in cell viability, and only the longest 30 min APPJ treatment affected slightly, with cell viability being around $80 \%$ which cannot be considered cytotoxic.

Flow cytometry analysis with annexin V- isopropidium iodide staining of SaOS-2 cells in contact with plasma-treated cell culture media was performed (Figure 7). Annexin V has high affinity for phosphatidylserine (PS) which is located on the cytoplasmic surface of normal viable cell membrane. In apoptotic cells, PS is translocated from the inner to the outer leaflet of the plasma membrane exposing PS to the external environment. PI is impermeant to live cells and apoptotic cells, whereas it penetrates the damaged membranes of necrotic cells which stain for red fluorescence, binding tightly to the nucleic acids in the cell. The results revealed that apoptosis was the main mechanism for 
cell death in the SaOS-2 cells in contact with APPJ-treated cell culture medium, with the dead cells being apoptotic (B2) and with increasing numbers of pre-apoptotic cells (B4) as time in contact with plasma-treated medium progresses (Figure $7 \mathrm{~d}$ ).

Fluorescence images also confirmed that the number of cells was lower when cultured in APPJ treated medium compared to untreated cells, which almost covered the surface after 72h (Figure 8). In addition, cells in APPJ treated medium showed a round shaped morphology at $24 \mathrm{~h}$ and $48 \mathrm{~h}$ whereas untreated cells were totally spread. After $72 \mathrm{~h}$, round cells were still observed in the APPJ treated condition. High magnification images showed that most cells after $24 \mathrm{~h}$ of exposition to APPJ treated medium presented short, faint and poorly organized actin stress fibers (Figure 8b; insets in red) compared to the well-defined cytoskeleton filaments observed in untreated cells. However, after $48 \mathrm{~h}$ and 72h the number of cells presenting well-defined cytoskeletal organization moderately increased in the APPJ treated medium situation although it was dramatically lower than the untreated cells. Focal adhesions were also compromised after 24h of exposition to APPJ treated medium, where cells exhibited fewer number of vinculin-stained focal adhesions as well as shorter length, size and thickness compared to untreated samples (Figure 8b; insets in green). Noteworthy, this behavior was also observed after $48 \mathrm{~h}$ and 72h.

\section{Discussion}

The particular characteristics of the bone niche prompt to investigate the potential of atmospheric pressure plasmas for treatment of bone cancers. Herein, we have shown that a Helium APPJ has direct cytotoxic effects on bone osteosarcoma cells (Figure 5) and 
this effect seems to be mediated by liquids, as comparable effects were found following contact of SaOS-2 cells with APPJ treated cell culture medium (Figure 6a).

The effects found in cells have to be related with the particular characteristics conferred by plasma to the liquid medium, and that is in turn, related to the characteristics of the plasma itself. Therefore, the discharge characteristics are a relevant issue when considering their effects on biological targets or liquids. Here, the APPJ length showed clear dependence with the He flow rate in ambient air (Figure 1), progressively increasing until around $6 \mathrm{~L} / \mathrm{min}$ where maximum jet length is reached. As reported by Lu et al. ${ }^{21}$ this can be ascribed to the change in the gas flow regime from laminar to turbulent, as the jet length increases with flow rate in the laminar flow regime and decreases upon transition to the turbulent flow.

The presence of a sample at a certain distance below the jet, the sample physical state and composition can change jet shape, jet length, its discharge parameters (voltage, current, power, electromagnetic field) and plasma parameters (concentrations of plasma species) ${ }^{20}$. This can clearly be observed by the increased emission intensity and jet shape in our experiments (Figure 1), as well as in the different optical emission intensity profiles of the emitting plasma species with the free jet (Figure 3a) or during treatment of cell culture media (Figure 3b).

Of course, the modified APPJ and discharge parameters subsequently have an impact on the liquid treatment. The reason for the reciprocal influence, in the case of single electrode plasma jets, is the fact that the sample itself acts like a second electrode and the discharge is actually running between the high voltage electrode and the treated sample surface ${ }^{20}$. 
The main species detected by OES (Figure 2) were Helium, $\bullet \mathrm{OH}, \mathrm{N}_{2} 2^{\text {nd }}$ positive, $\mathrm{N}_{2}{ }^{+} 1^{\text {st }}$ negative, and monoatomic O•. During APPJ treatment of liquid cell culture medium an increase in certain emission line intensities was recorded. In particular, the maximum intensity was recorded at $2 \mathrm{~L} / \mathrm{min}$ for $\mathrm{N}_{2} 2^{\text {nd }}$ positive band with line at $337 \mathrm{~nm}$. The other species detected moderately increased with gas flow rate. For instance, the increase of - $\mathrm{OH}$ intensity is the gas composition change i.e. humidity increase in the jet surroundings due to the aqueous sample. According to Lukes et al. ${ }^{22}$, the main source of $\bullet \mathrm{OH}$ radicals in the gas phase discharge is through the electron impact of $\mathrm{H}_{2} \mathrm{O}$ molecules in the water vapor above and near the water surface.

The interaction of the APPJ generated species directly at the gas-liquid interface and through post-discharge processes in plasma-activated liquid leads to the formation of a variety of reactive species. In particular, not only the gas phase, but also the components of the liquid media determine the species generated. In this work the progressive formation of $\mathrm{H}_{2} \mathrm{O}_{2}, \mathrm{NO}_{2}{ }^{-}$and $\mathrm{NO}_{3}{ }^{-}$was recorded in McCoy's (Figure 4) and advDMEM (Supplementary material) cell culture media with APPJ treatment time with similar results in both media for the nitrites and slightly higher $\mathrm{H}_{2} \mathrm{O}_{2}$ values in advDMEM. The distance between the APPJ capillary and the surface of the liquid media did not yield significant differences in the concentration of species generated, being time a more relevant parameter. While conventional cell culture media are usually buffered, in this work we avoided adding buffers to avoid masking potential effects of the APPJ. Even so, no significant changes were recorded in $\mathrm{pH}$, while conductivity tended to increase due to the generation of charged species in the media and solvated electrons ${ }^{23}$.

In addition to the species measured here $\left(\mathrm{H}_{2} \mathrm{O}_{2}, \mathrm{NO}_{2}{ }^{-}\right.$and $\left.\mathrm{NO}_{3}{ }^{-}\right)$, the creation of many others can be expected through contact of liquid with atmospheric plasma, especially 
considering the complex composition of the cell culture media evaluated that includes proteins, sugars, etc. All these compounds can react with the plasma and post-discharge gas phase species leading to a number of reactions. In particular, while positive and negative ions from the post-discharge largely combine or neutralize prior to diffusing into the liquid, lifetimes of neutral radicals are much longer, so that these reactive species are able to diffuse into the water without significant losses ${ }^{21}$. For instance, in plasma-treated aqueous systems different reactive species were detected ${ }^{10}$; Short-lived transient species (peroxynitrite $\mathrm{ONOO}^{-}$, hydroxyl radical $\bullet \mathrm{OH}$, nitrogen dioxide $\bullet \mathrm{NO}_{2}$, nitric oxide $\bullet \mathrm{NO}$ radicals and superoxide $\mathrm{O}_{2}^{-}$) and long-lived chemical products (ozone $\mathrm{O}_{3}$, hydrogen peroxide $\mathrm{H}_{2} \mathrm{O}_{2}$, nitric acid $\mathrm{HNO}_{3}$, nitrous acid $\mathrm{HNO}_{2}$ ) are formed in water following a plasma discharge, their density and concentration depending on the gas atmosphere and on the $\mathrm{pH}$ of plasma-treated water.

Cancer therapy is one of the most promising applications of atmospheric pressure plasmas, as their efficacy was demonstrated in vitro and in vivo for various cancer types of soft tissues (lung, bladder, breast, head, neck, brain or skin). Herein hard tissue cancer was in focus, so direct treatment of bone cancer cells (SaOS-2) cultured on wells and covered with cell culture medium (Figure 5) was performed as described before. As a result of He APPJ treatment, clear effects were observed; initially both cancer cells and healthy cells exhibited similar viability (around 65\% in all treatment conditions $24 \mathrm{~h}$ following the treatment). Then, while cancer cells were not able to recover after $72 \mathrm{~h}$ of culture and even displayed further viability decrease (down to $45 \%$ of cell viability for the longest treatment), hMSCs recovered showing 100\% viability and $1.5 \mathrm{~min}$ APPJ treated HObs even showed higher proliferation than the control. All this indicated a selective cytotoxicity of APPJ in the direct treatment. These cytotoxic effects might be mediated by liquids, as in the biological environment cells are covered by physiological 
liquids, and in some cases, plasma treatment of cell culture medium or liquid medium was an effective anti-tumor fluid, both in vitro and in vivo ${ }^{24,25}$.

Our results confirm this point: in indirect treatments, when APPJ-treated cell culture medium was put in contact with bone cancer cells, two effects could be highlighted; Firstly, depending on the APPJ treatment time, cell viability decreased markedly (between $90 \%$ and $20 \%$ at $24 \mathrm{~h}$ as a function of plasma treatment time) (Figure 6a). This decrease in cell viability is comparable to that found in other studies focused also on osteosarcoma but following chemotherapeutic approaches ${ }^{26,27}$. Secondly, the treatment had delayed effects, as live cells were continuously decreasing after contact with the treated liquid. In this way, in certain conditions as found for example for 30 min of APPJ liquid treatment, complete bone cancer cell death is obtained. Interestingly, healthy hMSCs, treated in the same condition were completely viable and unaffected by the treated liquid, even after 24h, contrary to what happened with the direct plasma treatment. HObs in contact with APPJ treated liquid remained essentially unaffected, with only a minor decrease in cell viability with the longest treatment of 30 min which could not be considered cytotoxic. This highlights one of the advantages of this indirect treatment with APPJ treated medium, namely, it avoids the effects of electric fields, UV light, etc. that could have damaging effects on healthy cells, and only allows long living APPJ generated reactive species or probably metastable species produced in reactions of the plasma with the liquid to interact with the cells.

In fact, the ROS and RNS generated in the liquid media can promote oxidative stress and trigger different signaling pathways in cells. ROS \& RNS have been described as tumorigenic by virtue of their ability to induce cell proliferation and migration sustaining tumor progression ${ }^{28}$. However, their concentrations regulate the switch between cell survival and death. Thus, whereas moderate concentrations of ROS \& RNS play a role as 
regulatory mediators in signaling processes, including signal transduction from membrane receptors (e.g. maturation of focal adhesions), high or excessive ROS \& RNS concentrations (i.e. oxidative stress) induce apoptotic cell death in various cell types. To investigate this point, cells were stained with Annexin V, a biochemical marker of apoptosis, and propidium iodide (PI) a marker of cell membrane integrity (Figure 7). Dead apoptotic cells display red and green staining on B2 corner of flow cytometry dot plots. As can be observed in Figure $7 \mathrm{~b}$ and d, APPJ treatment elicited the appearance of a cell population that was Annexin V positive / PI negative, and therefore can be considered as pre-apoptotic. This effect progressed with cell incubation time in contact with the APPJ treated medium. Therefore, the plasma doses employed in this work lead to apoptosis of the bone cancer cells, which is also in agreement with previous results of other authors ${ }^{29}$. This was observed for instance for cells co-treated with ROS scavengers, where they observed mitigation of the apoptotic effect of the APPJ ${ }^{8}$. This suggested that the apoptotic effects of the APPJ may be mediated by ROS.

Microscopy observation after APPJ treatment showed that the cells exhibit round shape morphology with disorganized actin stress fibers and few focal adhesions (Figure 8). Dramatic changes in actin filament organization have been demonstrated to be linked to apoptosis pathways ${ }^{30}$. In fact, actin is a substrate for cleavage by caspases, the main effectors of apoptosis mechanisms ${ }^{31}$. Actin fragmentation results in cell rounding which involves the loss of focal contacts contributing to apoptosis. In the present study we observed that the plasma-treated medium has a dramatic effect on the actin cystoskeleton organization as well as on focal adhesion formation (Figure 8 and 8b) which may contribute to increase apoptosis. However, this effect was stronger at earlier incubation times (24h) suggesting that the some of the species generated and involved in actin fragmentation may disappear over time. 
From the results obtained in this work and from previous results of other authors, it is clear that the long-living reactive species are probably the main agent which caused the APPJ-cell interaction ${ }^{31,32}$. At the gas-liquid interface, plasma driven chemistry leads to the solvation of primary long living species along with solvated electrons, which results in the generation of secondary long living species with high abundances. Wende et al. ${ }^{25}$, pointed out that the indirect treatment retained the long lived ROS and RNS while reducing effects caused by inhomogeneous treatment. In our case, the indirect treatment of liquid could add to this advantage, since APPJ treated liquid with anticancer activity could potentially be injected in the bone tumor site with a minimally invasive approach. Moreover, this method could be applicable to different sites in the skeleton, when dealing with bone metastases.

\section{CONCLUSIONS}

Atmospheric pressure plasma jet generated different gaseous reactive oxygen species (ROS) and reactive nitrogen species (RNS) that were transmitted to the liquid phase through formation of secondary species such as peroxides, nitrates or nitrates, among others. This APPJ generated cocktail of reactive species exhibited lethal effects towards bone cancer cells in a selective manner, avoiding harmful effects for healthy mesenchymal stem cells. Treatment was effective both in direct treatment of cells or through indirect treatment of liquid medium which was added to cell cultures, which showed to die through apoptotic mechanisms. The lethal effects were shown to have delayed activity, and were possibly related to the generation of ROS and RNS in the liquid media. Further studies have to be performed to ascertain the molecular mechanisms involved in this effective selectivity towards bone cancer cells. 


\section{ACKNOWLEDGEMENTS}

Authors acknowledge the financial support of the COST Action TD1208, of MAT201565601-R project (MINECO/FEDER, EU) and of SGR2014 01333. This project has received funding from the European Research Council (ERC) under the European Union's Horizon 2020 research and innovation programme (grant agreement No 714793). CC acknowledges MINECO for the Ramon y Cajal fellowship. JGM thanks the support of the AGAUR of the Government of Catalonia (2014-PDJR-00014). Support for the research of MPG was received through the ICREA Academia Award for excellence in research, funded by the Generalitat de Catalunya. UC acknowledges financial support from NATO grant SPS.984555 and Slovenian Resarch Agency (ARRS). L. Thevenart is acknowledged for technical support.

\section{REFERENCES}

1. American Cancer Society. Cancer Facts \& Figures 2016. Cancer Facts Fig 2016. 2016;1-9.

2. Tavazoie SF, Alarcon C, Oskarsson T, Padua D, Wang Q, Bos PD, et al.

Endogenous human microRNAs that suppress breast cancer metastasis. Nature. Nature Publishing Group; 2008;451:147-52.

3. Driel M Van, Leeuwen JPTM Van. Cancer and bone : A complex complex. 2014;561:159-66.

4. Schlegel J, Köritzer J, Boxhammer V. Plasma in cancer treatment. 2013;1:2-7.

5. Graves DB. Reactive Species from Cold Atmospheric Plasma: Implications for Cancer Therapy. Plasma Process Polym. 2014;1-8.

6. Ratovitski EA, Cheng X, Yan D, Sherman JH, Canady J, Trink B, et al. Anticancer therapies of 21st century: Novel approach to treat human cancers using 
cold atmospheric plasma. Plasma Process. Polym. 2014. page 1128-37.

7. Schmidt A, Bekeschus S, von Woedtke T, Hasse S. Cell migration and adhesion of a human melanoma cell line is decreased by cold plasma treatment. Clin Plasma Med. 2015;3:24-31.

8. Ahn HJ, Kim K Il, Kim G, Moon E, Yang SS, Lee JS. Atmospheric-pressure plasma jet induces apoptosis involving mitochondria via generation of free radicals. PLoS One. 2011;6:6-12.

9. Park S-B, Kim B, Bae H, Lee H, Lee S, Choi EH, et al. Differential Epigenetic Effects of Atmospheric Cold Plasma on MCF-7 and MDA-MB-231 Breast Cancer Cells. PLoS One. 2015;10:e0129931.

10 Graham WG, Stalder KR. Plasmas in liquids and some of their applications in nanoscience. J Phys D Appl Phys. 2011;44:174037.

11 Oehmigen K, Hähnel M, Brandenburg R, Wilke Ch,Weltmann K-D,Von Woedtke Th.The role of acidification for antimicrobial application of atmospheric pressure plasma in liquids. Plasma Process Polym. 2010; 7, 250.

12 Tanaka H, Mizuno M, Ishikawa K, Nakamura K, Kajiyama H, Kano H, Kikkawa F, Hori M. Plasma-Activated Medium Selectively Kills Glioblastoma Brain Tumor Cells by Down-Regulating a Survival Signaling Molecule, AKT Kinase. Plasma Medicine. 2013; 1: 265-277.

13 Tanaka H, Mizuno M, Ishikawa K, Nakamura K, Utsumi F, Kajiyama H, Kano H, Maruyama S, Kikkawa F, Hori M. Cell survival and proliferation signaling pathways are downregulated by plasma-activated medium in glioblastoma brain tumor cells. Plasma Medicine 2014; 2: 207-220. 
14 Utsumi F, Kajiyama H, Nakamura K,Tanaka H, Mizuno M, Ishikawa K, Kondo H, Kano H, Hori M, Kikkawa F. Effect of Indirect Nonequilibrium Atmospheric Pressure Plasma on Anti-Proliferative Activity against Chronic Chemo-Resistant Ovarian Cancer Cells In Vitro and In Vivo. Plos One 2013 ; 8: e81576.

15 Utsumi, F, Kajiyama H, Nakamura K, Tanaka H, Hori M, Kikkawa F. Selective cytotoxicity of indirect nonequilibrium atmospheric pressure plasma against ovarian clear-cell carcinoma. SpringerPlus 2014; 3:398-407.

16 Torii K, Yamada S, Nakamura K, Tanaka H, Tanahishi K, et al. Effectiveness of plasma treatment on gastric cancer cells. Gastric cancer: official journal of the International Gastric Cancer Association and the Japanese Gastric Cancer Association 2014; 18: 635-643.

17 Hattori N, Yamada S, Torii K, Takeda S, Nakamura K, Tanaka H, et al. Effectiveness of plasma treatment on pancreatic cancer cells. International journal of oncology 2015; 47: 1655-1662.

18 Adachi T, Tanaka H, Nonomura S, Hara H, Kondo S, Hori M. Plasma-activated medium induces A549 cell injury via a spiral apoptotic cascade involving the mitochondrialnuclear network. Free radical biology \& medicine 2014; 79C: 2844.

19 Vandamme M, Robert E, Lerondel S, Sarron V, Ries D, Dozias S, Soblio J, Gosset D, Kieda C, Legrain B, Pouvesle JM, Pape AL. Int. J. Cancer 2012 ; 130: 21852194.

20 Zaplotnik R, Bišćan M, Kregar Z, Cvelbar U, Mozetič M, Milošević S. Influence of a sample surface on single electrode atmospheric plasma jet parameters. 
Spectrochim Acta - Part B At Spectrosc. 2015;103-104:124-30.

21 Lu X, Naidis G V., Laroussi M, Reuter S, Graves DB, Ostrikov K. Reactive species in non-equilibrium atmospheric-pressure plasmas: Generation, transport, and biological effects. Phys Rep. 2016;

22 Lukes P, Locke BR. Plasmachemical oxidation processes in a hybrid gas - liquid electrical discharge reactor. 2005;38:4074-81.

23 Rumbach P, Bartels DM, Sankaran RM, Go DB. The solvation of electrons by an atmospheric-pressure plasma. Nat Commun. 2015;6:7248.

24 Hori M, Mizuno M, Kikkawa F, Kajiyama H, Utsumi F, Nakamura K, et al. US2015/0030693 A1 Anti-tumor aqueous solution, anti-cancer agent, and methods for producing said aqueous solution and said anti-cancer agent. 2015.

25 Wende K, Reuter S, Von Woedtke T, Weltmann KD, Masur K. Redox-based assay for assessment of biological impact of plasma treatment. Plasma Process Polym. 2014;11:655-63.

26 Soares PIP, Sousa AI, Carvalho Silva J, Ferreira IMM, Novo CMM, Borges JP. Chitosan-based nanoparticles as drug delivery systems for doxorubicin: optimization and modelling. Carbohydrate Polym. 2016; 147: 304-312.

27 Zhou J, Gan M, Mao N, Zhu X, Shi Q, Yang H. Sensitization of Osteosarcoma cell line SaOS-2 to chemotherapy by downregulating Survivin. Archives Medical Res 2010; 41:162-169

26 Wiseman H, Halliwell B. Inflammatory Disease and Progression To Cancer. Biochem J. 1996;29:17-29. 
27 Graves DB. Oxy-nitroso shielding burst model of cold atmospheric plasma therapeutics. Clin Plasma Med. 2014;2:38-49.

28 Häcker G. The morphology of apoptosis. Cell Tissue Res. 2000;301:5-17.

29 Mashima T, Naito M, Tsuruo T. Caspase-mediated cleavage of cytoskeletal actin plays a positive role in the process of morphological apoptosis. Oncogene. 1999;18:2423-30.

30 Wende K, Straßenburg S, Haertel B, Harms M, Holtz S, Barton A, et al. Atmospheric pressure plasma jet treatment evokes transient oxidative stress in HaCaT keratinocytes and influences cell physiology. Cell Biol Int. 2014;38:41225. 


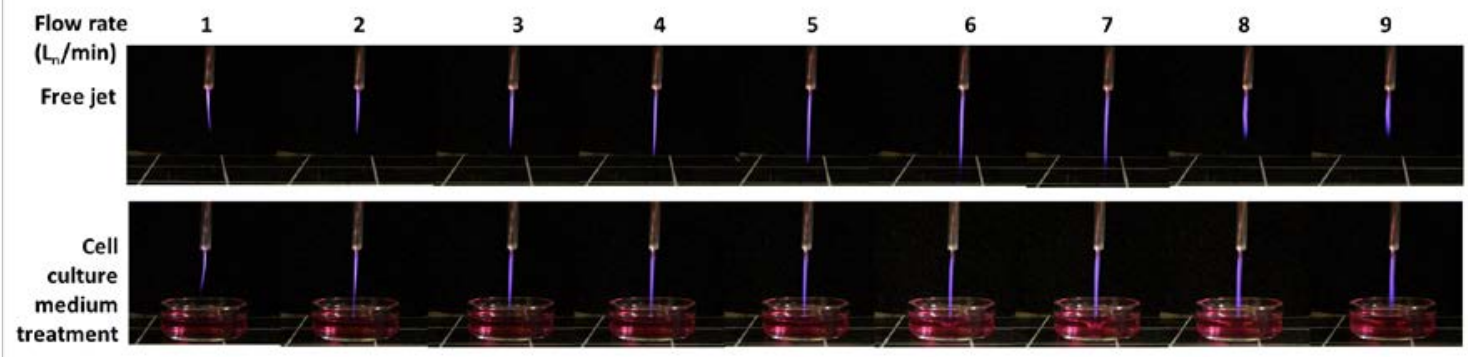

Figure 1. Photographs of the APP free jet (top) and during treatment of liquid media (bottom) at increasing gas flow rates (from left to right).

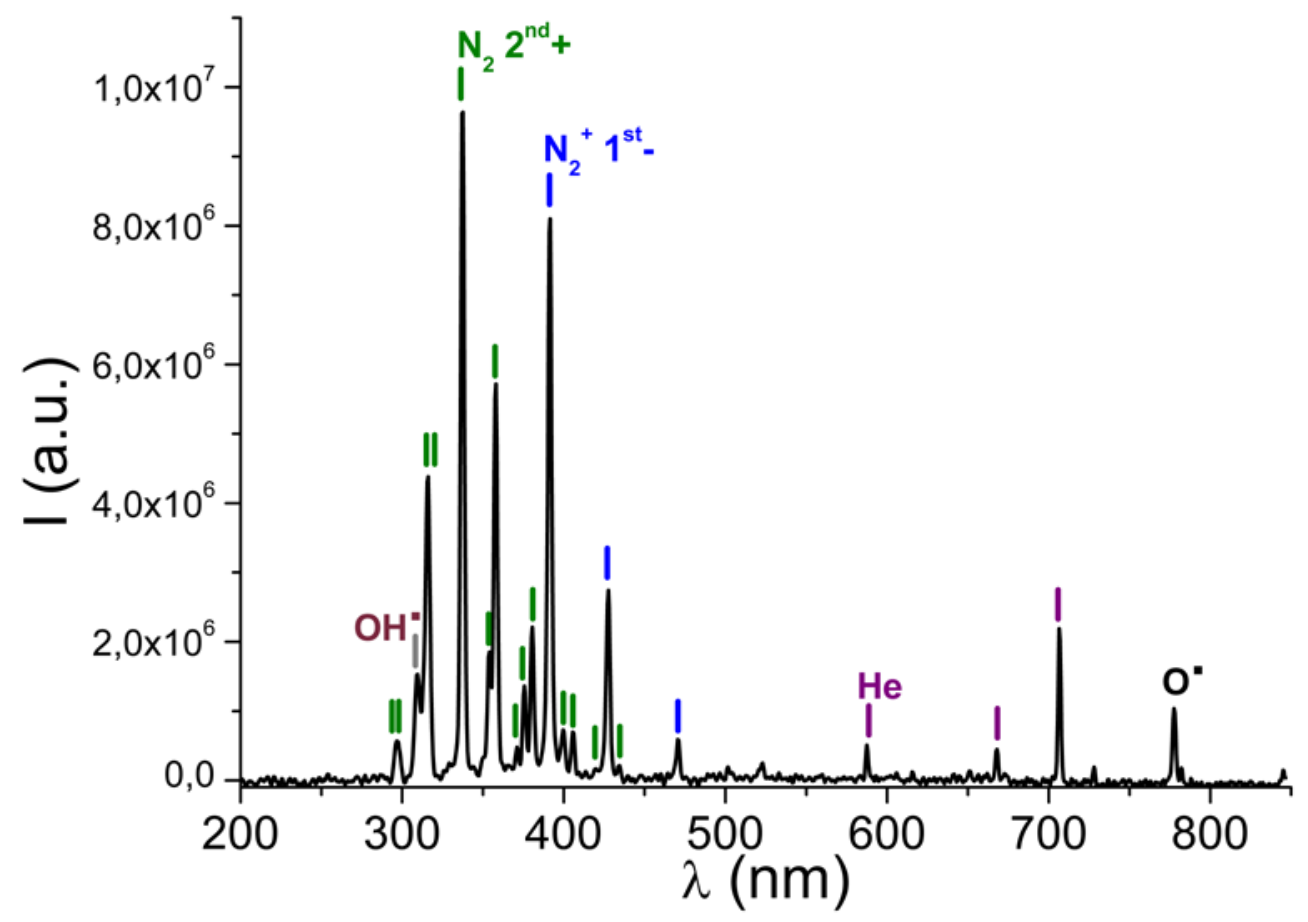

Figure 2. Representative optical emission spectra of the He APPJ at the distance of 10 $\mathrm{mm}$ from the tip of the capillary (presented for gas flow rate: $5 \mathrm{~L} / \mathrm{min}$ ) during treatment of McCoy's cell culture medium (at 20 mm distance). 


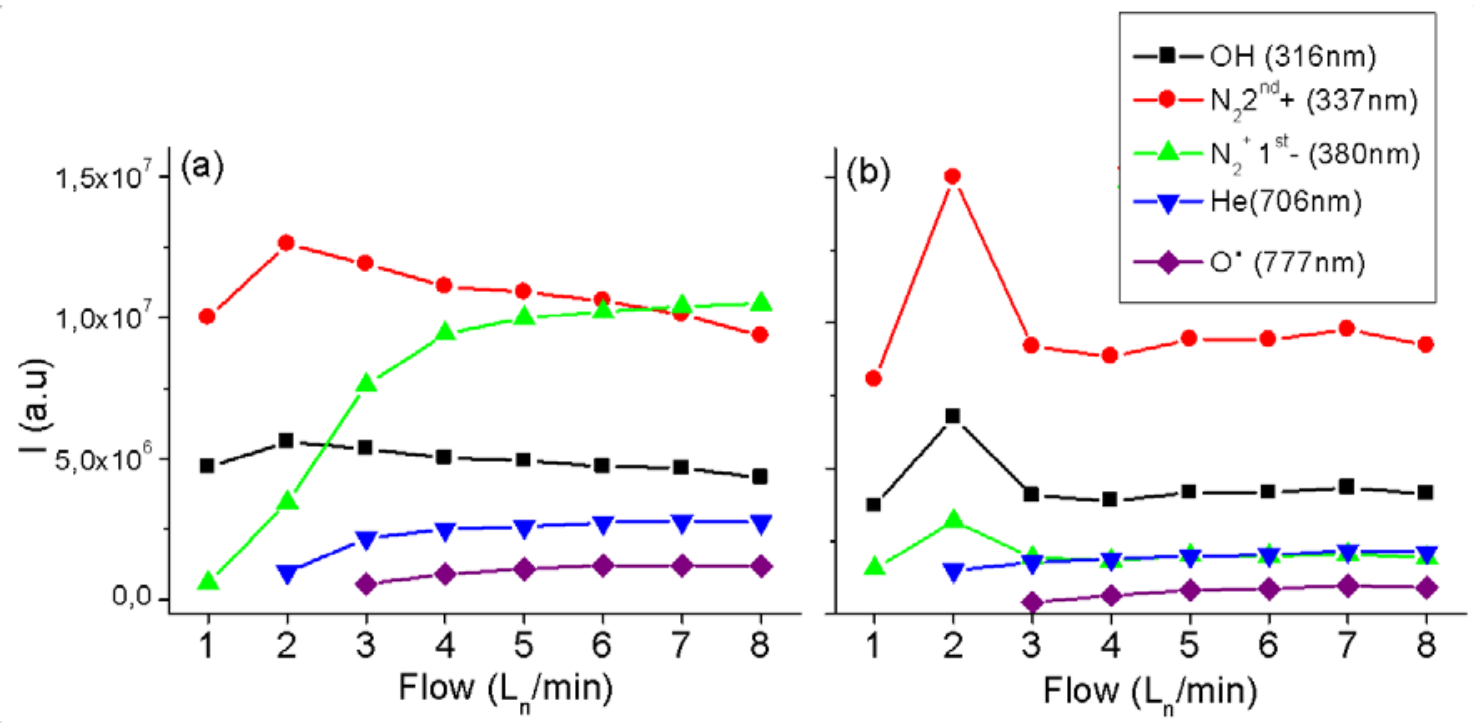

Figure 3. Dependence of the optical emission intensity of the different plasma species in the post-discharge (measured $10 \mathrm{~mm}$ from the tip of the jet) (a) or in presence of cell culture liquid media (placed at a distance of $20 \mathrm{~mm}$ ) (b) with increasing He flow rates during APPJ treatment.
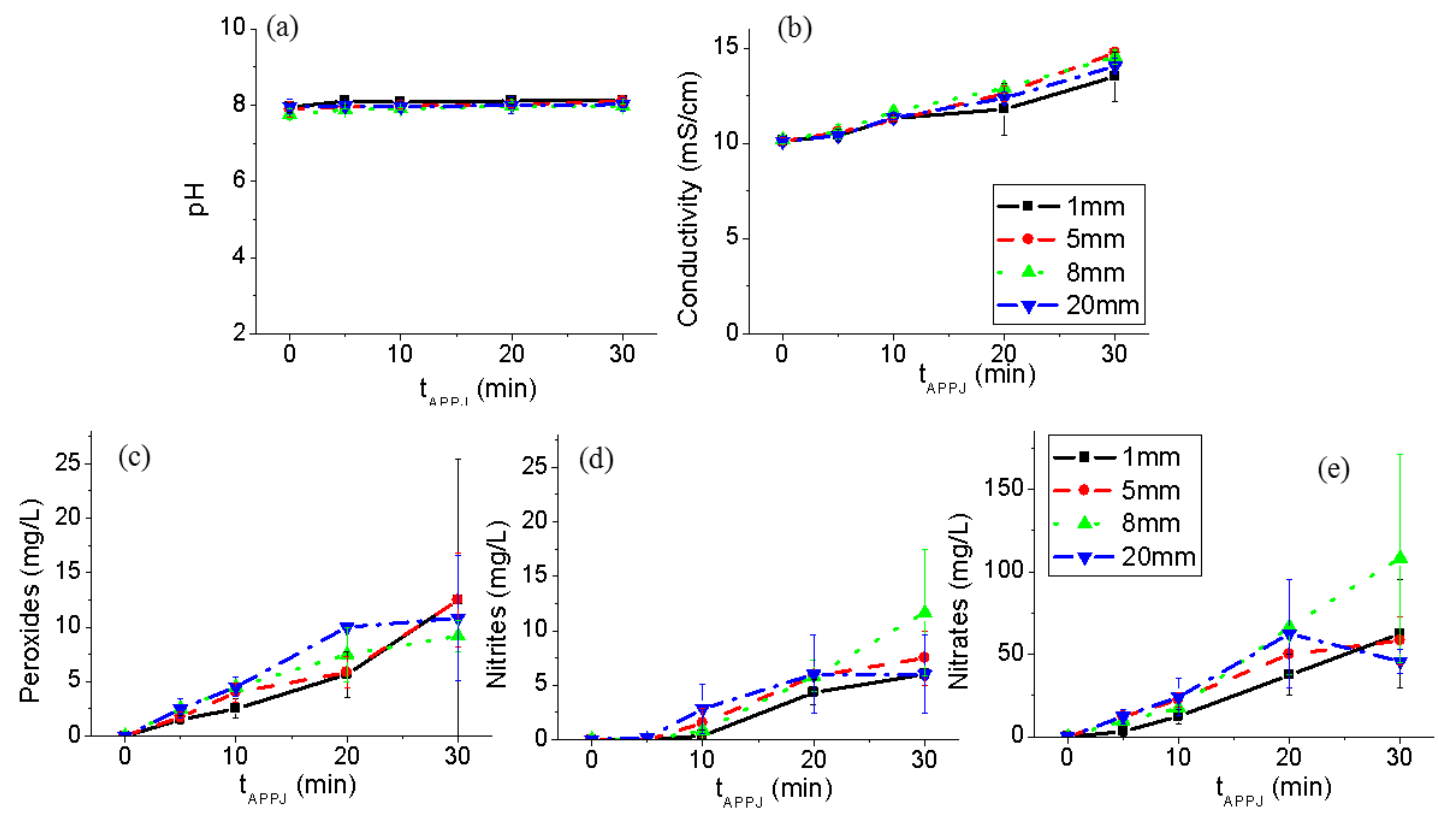

Figure 4. Influence of He atmospheric pressure plasma treatment time (tAPPJ) $(5 \mathrm{~L} / \mathrm{min})$ on different parameters of McCoy's cell culture media at different distances capillaryliquid. pH (a), conductivity (b), peroxides (c), nitrites (d), nitrates (e). 


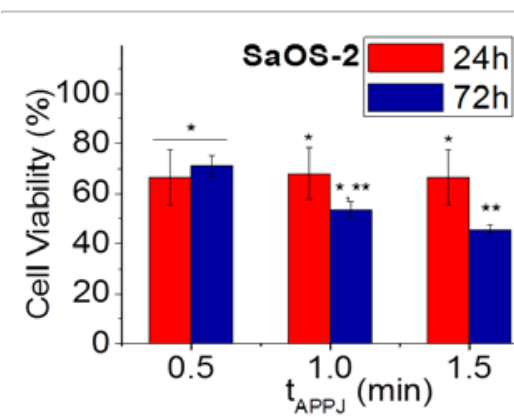

(a)

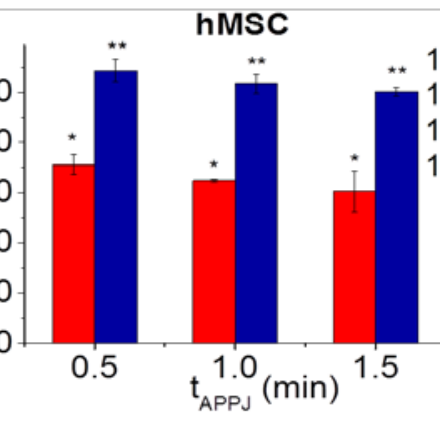

(b)

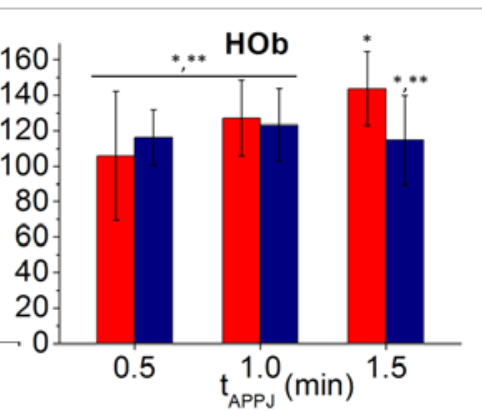

(c)

Figure 5. Cytotoxic effects of direct plasma treatment on human cells (a) SaOS-2 cells, (b) hMSCs and (c) HObs at different incubation times. *, ** indicate statistically significant differences.

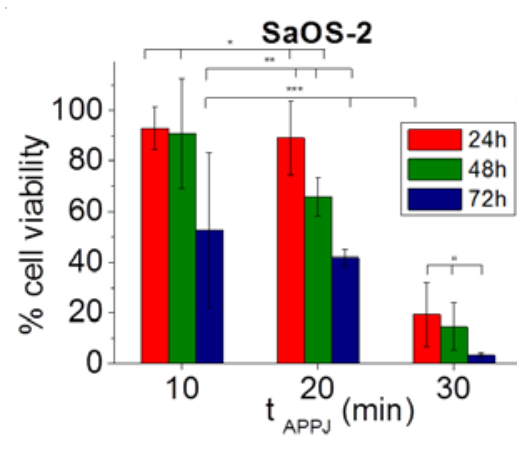

(a)

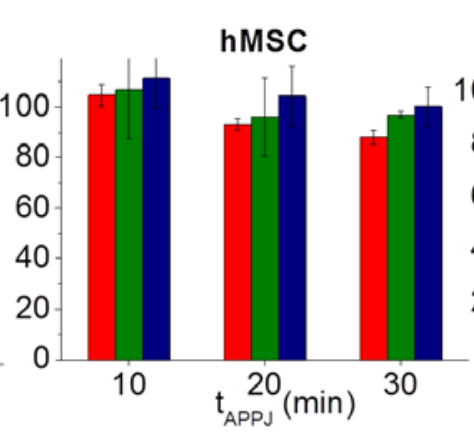

(b)

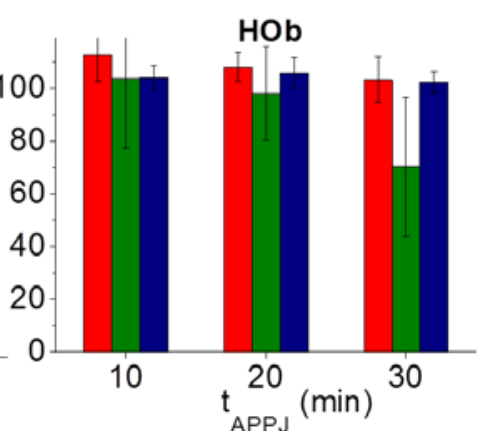

(c)

Figure 6. Cytotoxicity of plasma treated cell culture media (McCoy and advDMEM, respectively) on human cells (a) SaOS-2 cells, (b) hMSCs and (c) HObs at different incubation times. $*, * *, * * *$ indicate statistically significant differences. No statistically significant differences in (b) and (c). 

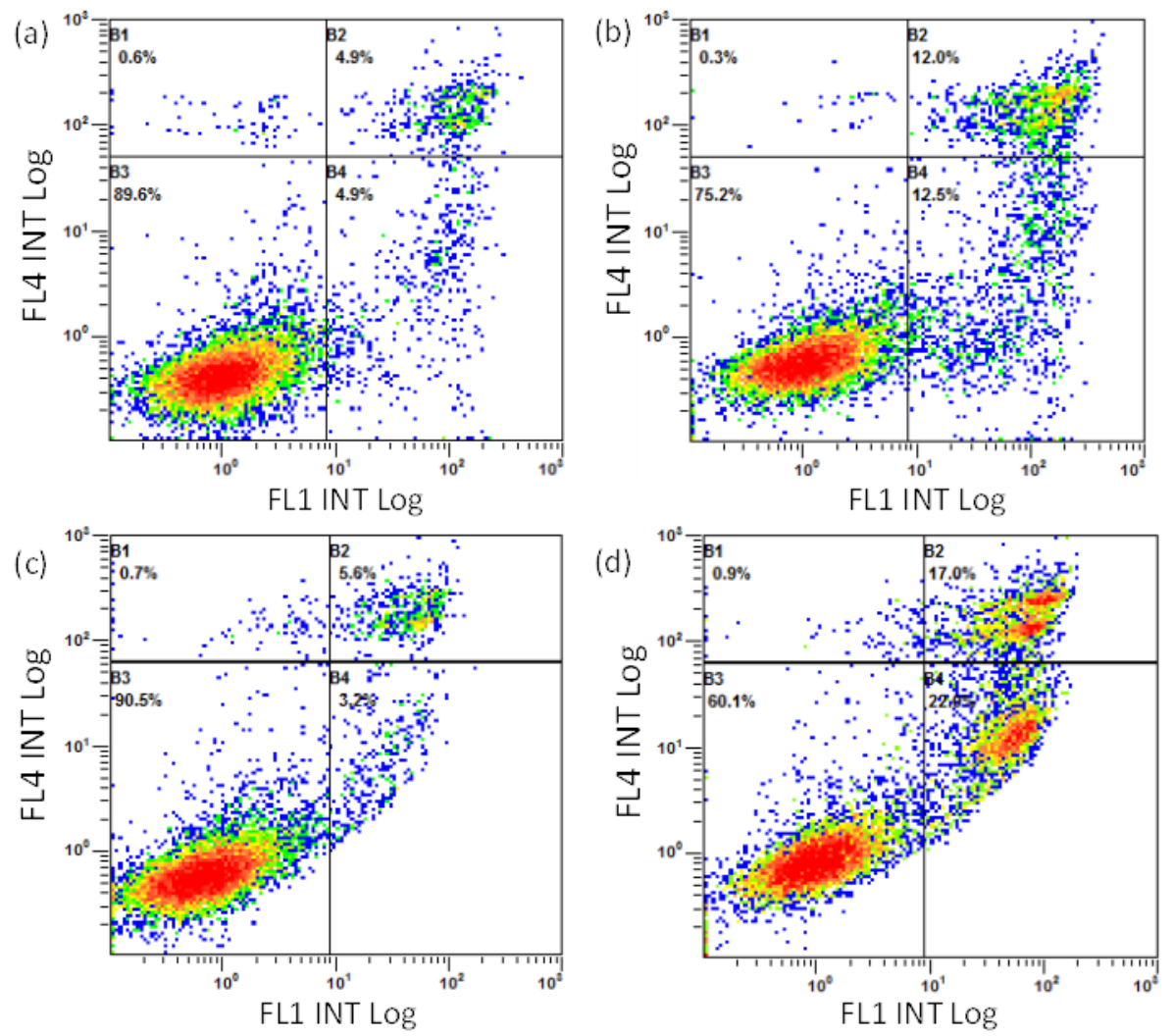

Figure 7. Representative flow cytometry images (annexin- propidium iodide) of SaOS-2 cells cultured in plasma-treated culture media (30 min). (a) untreated and (b) plasma treated after 24 h, (c) untreated and (d) plasma treated after $72 \mathrm{~h}$ of cell culturing. 

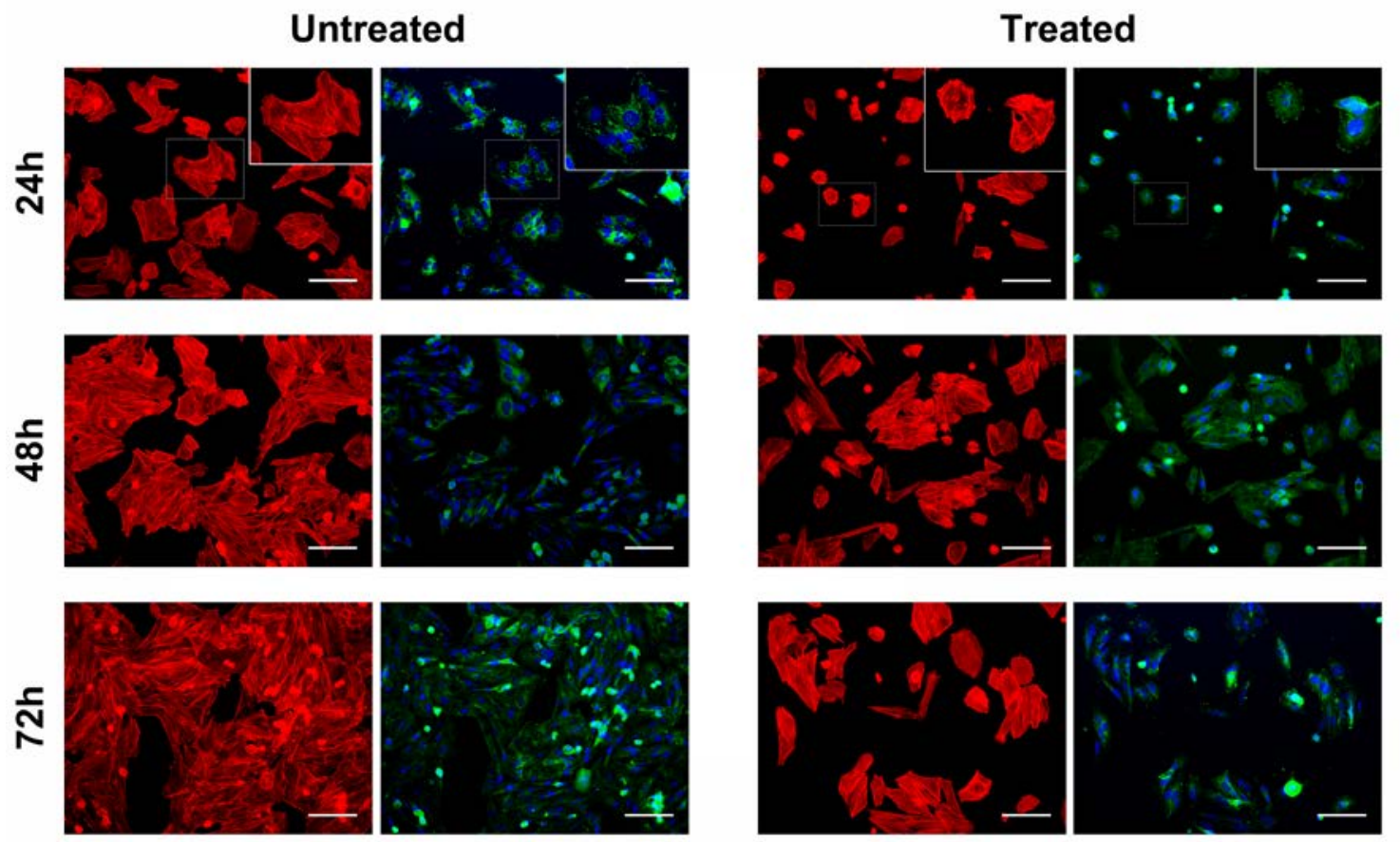

Figure 8. Representative fluorescence images of SaOS-2 cells cultured in untreated (a) or plasma-treated culture media (30 min) (b) and stained for actin (red), vinculin (green) and nuclei (blue). 20X magnification images (scale bar $=100 \mu \mathrm{m}$ ). Insets show magnified cells for better visualization of actin filaments and vinculin adhesion staining. 